\title{
Headache attack followed by rapid disease progression in pediatric moyamoya disease—how should we manage it?
}

\author{
Sandra Vuignier • Naoki Akioka • Hideo Hamada • \\ Daina Kashiwazaki $\cdot$ Satoshi Kuroda
}

Received: 26 June 2013 / Accepted: 24 March 2014 / Published online: 1 April 2014

(C) Springer-Verlag Berlin Heidelberg 2014

\begin{abstract}
Case report A 4-year-old female was presented at our hospital with frequent right frontal headache attack. She was diagnosed with moyamoya disease and was conservatively followed up. One year later, the frequency of headache gradually decreased. However, follow-up MR imaging revealed that the disease stage markedly progressed in the right side and cerebral infarction occurred in the temporal lobe with atrophy of the right frontal lobe. She underwent direct and indirect revascularization on the right side.

Conclusion Aware of this case, we would like to emphasize that headache may be one subtype of ischemic attacks and require frequent MR follow-up to see the disease course. If there is any sign of disease progression, immediate surgical intervention should be indicated to avoid irreversible brain damage.
\end{abstract}

Keywords Moyamoya disease $\cdot$ Headache $\cdot$ Cerebral revascularization $\cdot$ Natural history $\cdot$ Disease progression

\section{Introduction}

Moyamoya disease is an uncommon cerebrovascular disease characterized by progress stenosis in the terminal internal carotid arteries (ICAs) and their main branches, which results in the formation of fine vascular networks at the base of the

S. Vuignier $\cdot$ N. Akioka $\cdot$ H. Hamada $\cdot$ D. Kashiwazaki $\cdot$

S. Kuroda $(\bowtie)$

Department of Neurosurgery, Graduate School of Medicine and

Pharmaceutical Science, University of Toyama, 2630 Sugitani,

Toyama 930-0194, Japan

e-mail: skuroda@med.u-toyama.ac.jp

S. Vuignier

Division of Neurosurgery, University Hospital of Geneva,

Geneva, Switzerland brain (moyamoya vessels) to compensate cerebral ischemia. Interestingly, moyamoya disease has two age distribution peaks at around 5 and 40 years. Most pediatric patients exhibit transient ischemic attack (TIA) or ischemic stroke. Headache and involuntary movements are also serious symptoms associated with pediatric moyamoya disease $[1,2]$. Although the disease is rare, it is an important cause of ischemic stroke in children, and recently, it has become more widely recognized worldwide as a cause of pediatric cerebrovascular events. Thus, moyamoya disease should be considered in any child who presents with only headache attack.

In this report, we describe a pediatric case that presented with headache attacks due to moyamoya disease. During 1 year of follow-up, rapid disease progression led to cerebral infarction associated with intellectual impairment.

\section{Case}

A 4-year-old female was presented at our hospital with frequent episodes of right frontal headache. She complained of it when she waked up in the morning and sometimes vomited. Physical and neurological examinations revealed no definite abnormality. Laboratory examinations, including erythrocyte sedimentation, somatic antibodies, and leptospiral antibodies, were all negative and ruled out any other underlying diseases. MR imaging (MRI) demonstrated no parenchymal lesion, but MR angiography (MRA) showed significant stenosis at the terminal portion of the right ICA and moyamoya vessels at the base of brain. There were no vascular lesions in the left side (Fig. 1). Therefore, we diagnosed as unilateral moyamoya disease. Except for the headache attacks, she was asymptomatic and her conditions seemed stable, so follow-up MR examination was scheduled 1 year later. One year later, she came back to the hospital for the follow-up. The frequency of headache attacks gradually decreased. However, MRA 
revealed that the disease stage on the right side markedly progressed from stage 2 to stage 4 on Suzuki's grading. Right posterior cerebral artery was also involved. MRI demonstrated that cerebral infarction occurred in the right temporal lobe. There was also cerebral atrophy in the right frontal lobe (Fig. 1). 123I-IMP single photon emission computed tomography (SPECT) showed a marked reduction of cerebral blood flow in the right cerebral hemisphere. The intelligence quotient (IQ) was impaired. Therefore, she underwent superficial temporal artery to middle cerebral artery (STA-MCA) anastomosis combined with indirect bypass, encephalo-duro-myoarterio-pericranial synangiosis (EDMAPS) [3, 4]. The frontal branch of STA was anastomosed to the prefrontal artery of MCA (0.8 $\mathrm{mm}$ in diameter). Temporary clamping time of MCA was $20 \mathrm{~min}$. The patency of STA-MCA anastomosis was confirmed, using near infrared indocyanine green (ICG) videoangiography during surgery. The dura mater, temporal muscle, and frontal pericranium were used as the donor tissues for indirect bypass. Postoperative course was uneventful. She was discharged without any perioperative complications. During follow-up period, her headache attack almost disappeared. Repeated cerebral angiography revealed well-developed collaterals through both direct and indirect bypass (Fig. 2).

\section{Discussion}

Headache was not considered as an important clinical sign of pediatric moyamoya disease a decade ago. With the accumulation of clinical data, however, headache attack is now recognized as one of important initial symptoms [5-7]. Yamashiro et al. reported clinical feature of 10 Japanese children with moyamoya disease. The most common initial manifestations were headache in four cases $(40 \%)$ and motor deficit and convulsion in three cases (30\%) [7]. Battistella and Carollo described 34 Italian patients suffering from moyamoya disease. The early clinical symptoms consisted of TIA and/or stroke (44\%), recurrent migraine-like headache (20\%), seizure (18\%), and hemorrhage (3\%) [8]. Matsushima et al. reported that about ones-third of pediatric patients complained of headache at initial presentation. Headache significantly affected their activity of daily life (ADL) in more than $60 \%$ of them. Majority of headache was localized in the frontal (40\%) or temporal region (25\%) [9]. Seol et al. also reported that headache was documented in $44(21.6 \%)$ of 204 pediatric patients with moyamoya disease. They also described that clinical course of headache in pediatric moyamoya disease had the following features: (1) a coexisting stage of headache and TIA; (2) the second stage of headache only; and (3) the final stage of improvement or disappearance of headache [6]. In fact, the frequency of headache gradually decreased during 1 year in the present case. Very recently, Kawabori et al. also reported the incidence and clinical features in pediatric moyamoya disease. Thus, headache attack was documented in 11 (38\%) of 29 pediatric patients. Severe disabling headache often develops in the frontal or temporal region in the morning but spontaneously resolves within 3 to $4 \mathrm{~h}[5]$.

Underlying mechanisms of headache in pediatric moyamoya disease is still unclear. Headache may arise from hypoperfusion-induced activation of pain-sensitive structures such as both intracranial and extracranial vasculatures, dura mater, and orbital and nasal cavities. Concomitantly, other mechanisms such as dilatation of the meningeal collaterals stimulating dural nocireceptors and ischemia-induced
Fig. 1 T2-weighted MR imaging $(\mathbf{a}, \mathbf{c})$ and MR angiography $(\mathbf{b}, \mathbf{d})$. At initial presentation, there was no parenchymal lesion in the brain (a). The occlusive lesion in the right carotid termination was moderate (Suzuki's stage 2, b). One year later, however, cerebral infarction developed in the right temporal lobe. Cerebral atrophy was noted in the right frontal lobe (c). Disease stage markedly progressed in the right carotid termination to Suzuki's stage 4 . The flow signal in the right posterior cerebral artery also decreased (d)
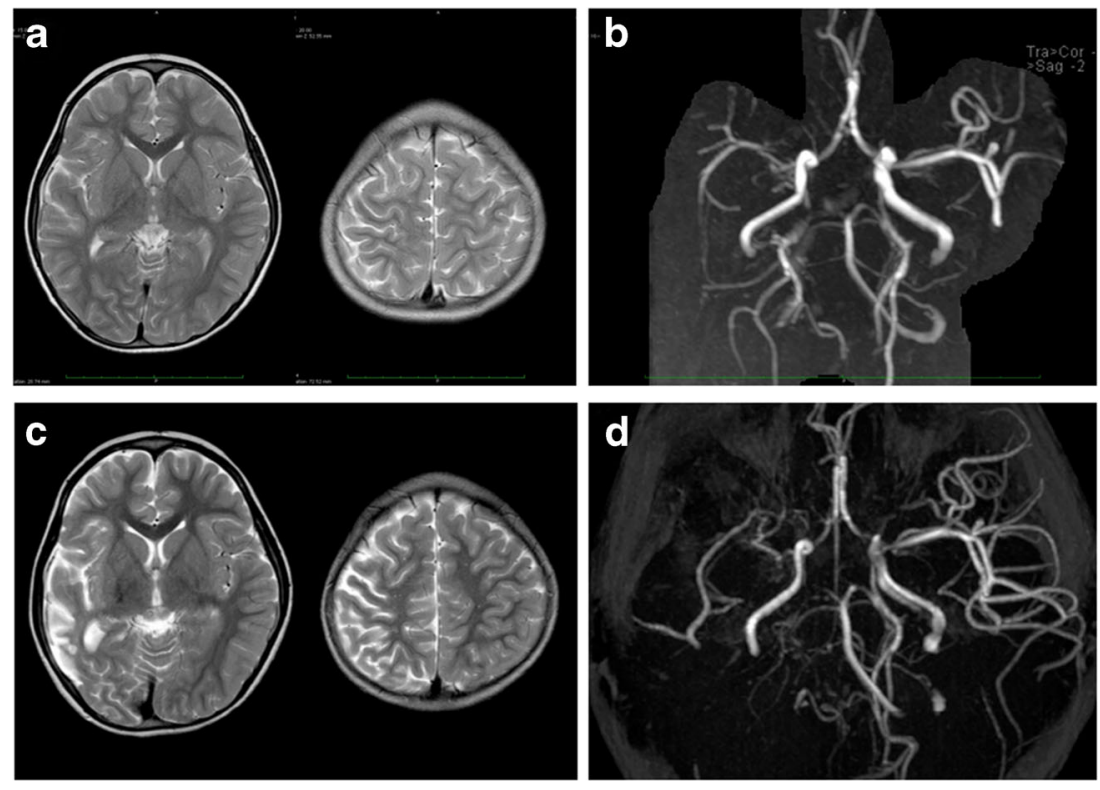


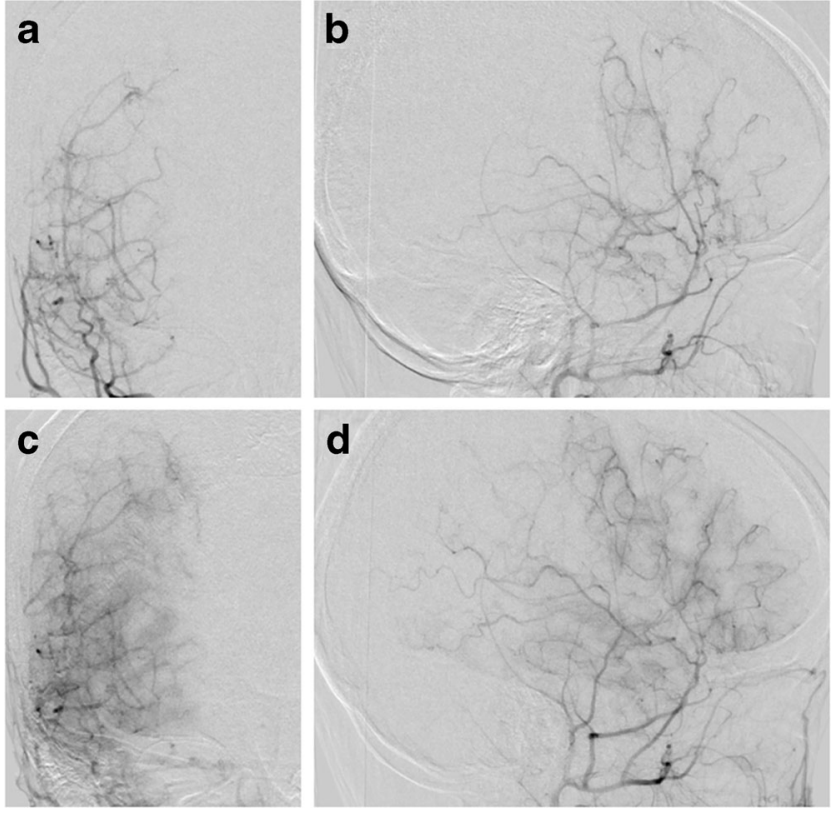

Fig. 2 Towne's view (a, c) and lateral view of postoperative right external carotid angiogram $(\mathbf{b}, \mathbf{d})$. Panels $\mathbf{a}$ and $\mathbf{b}$ represent early arterial phase, and panels $\mathbf{c}$ and $\mathbf{d}$ represent late arterial phase. They demonstrate that both direct and indirect bypass widely provide collateral blood flow to the right cerebral hemisphere

lowering of the migraine threshold have also been suggested [6]. Therefore, headache may develop as a symptom of TIA in pediatric moyamoya disease. Indeed, headache attack in pediatric patients is closely related to more advanced disease stage and also to decrease in cerebral blood flow and its reactivity to acetazolamide [5]. In the pediatric cases with atypical attacks of migraine and/or absence of a family history of migraine, therefore, a detailed investigation should be performed to detect underlying vascular diseases such as moyamoya disease.

Furthermore, there are a limited number of studies that denote the effect of surgical therapy on headache in pediatric moyamoya disease. Matsushima et al. reported that headache improved or disappeared in about $75 \%$ of pediatric patients after encephalo-duro-arterio-synangiosis (EDAS) [9]. However, Seol et al. concluded that more than $60 \%$ of pediatric patients still complained of headache even after EDAS [6]. Indeed, EDAS may provide no or little collateral blood flow to the frontal region where headache attacks often occur. Therefore, the impact of EDAS on headache attack is still controversial in pediatric moyamoya disease. On the other hand, very recent report has shown that STA-MCA anastomosis combined with EDMAPS markedly improves cerebral hemodynamics and resolves headache attacks in all of 11 pediatric patients with moyamoya disease, suggesting the importance of surgical design through a large craniotomy covering the frontal area [5]. It is still obscure whether surgical revascularization would be beneficial for pediatric patients with only headache attack due to moyamoya disease. However, there is the possibility that surgical revascularization at initial presentation may possibly prevent cerebral infarction in the present case. Further studies are warranted to clarify this issue. Welldesigned surgical treatment should be indicated without delay, at least when the pediatric patients with only headache due to moyamoya disease shows disease progression or deterioration of cerebral hemodynamics on follow-up examinations. Future clinical testing would be warranted to resolve this issue.

In the present case, disease stage markedly progressed during 1 year of follow-up, causing irreversible brain damage. Occlusive lesions in the carotid terminations often worsen in pediatric moyamoya disease $[10,11]$. In fact, Fukuyama and Umezu reported that occlusive lesions progressed in about two-thirds of hemispheres on repeat cerebral angiography with a mean interval of about 3 years in pediatric moyamoya disease [12]. Therefore, the radiological course in the present case may be natural, although the incidence of disease progression during 1 year is still unknown. Based on these observations, follow-up MR examination should be planned with shorter interval, for example, every 3 to 6 months. In addition, it is well known that functional and/or intellectual outcomes are poor in pediatric patients presenting with ischemic stroke due to moyamoya disease [13-15]. To prevent the occurrence of cerebral infarction, therefore, regular imaging studies should also be planned with shorter intervals in the conservatively treated children with moyamoya disease, because MRI and MRA studies enable it to accurately detect disease progression at outpatient clinic [16]. Surgical revascularization should also be indicated in the cases that show rapid disease progression in order to prevent permanent neurological and cognitive deficits.

In summary, we describe a case of pediatric moyamoya disease that presented with headache attacks at initial presentation. During 1 year of follow-up, a rapid disease progression caused cerebral infarction associated with cognitive impairment. We are convinced that a closer radiological monitoring would identify the disease progression more promptly and prevent permanent neurological sequelae due to ischemic stroke by indicating surgical revascularization.

Acknowledgments This study was partly supported by a grant from the Research Committee on Moyamoya Disease sponsored by the Ministry of Health, Labor, and Welfare of Japan.

\section{References}

1. Kuroda S, Houkin K (2008) Moyamoya disease: current concepts and future perspectives. Lancet Neurol 7:1056-1066

2. Suzuki J, Takaku A (1969) Cerebrovascular "moyamoya" disease. Disease showing abnormal net-like vessels in base of brain. Arch Neurol 20:288-299 
3. Kuroda S, Houkin K, Ishikawa T, Nakayama N, Iwasaki Y (2010) Novel bypass surgery for moyamoya disease using pericranial flap: its impacts on cerebral hemodynamics and long-term outcome. Neurosurgery 66:1093-1101, discussion 1101

4. Kuroda S, Houkin K (2012) Bypass surgery for moyamoya disease: concept and essence of surgical technique. Neurol Med Chir (Tokyo) $52: 287-294$

5. Kawabori M, Kuroda S, Nakayama N, Hirata K, Shiga T, Houkin K, Tamaki N (2013) Effective surgical revascularization improves cerebral hemodynamics and resolves headache in pediatric moysamoya disease. World Neurosurg 80:612-619

6. Seol HJ, Wang KC, Kim SK, Hwang YS, Kim KJ, Cho BK (2005) Headache in pediatric moyamoya disease: review of 204 consecutive cases. J Neurosurg 103:439-442

7. Yamashiro Y, Takahashi H, Takahashi K (1984) Cerebrovascular moyamoya disease. Eur J Pediatr 142:44-50

8. Battistella PA, Carollo C (1997) Clinical and neuroradiological findings of moyamoya disease in Italy. Clin Neurol Neurosurg 99(Suppl 2):S54-S57

9. Matsushima Y, Aoyagi M, Nariai T, Nojiri T, Ohno K (2000) Headache in pediatric moyamoya patients: pre- and postoperative changes. Nerv Syst Child (Jpn) 25:442-447
10. Ezura M, Yoshimoto T, Fujiwara S, Takahashi A, Shirane R, Mizoi K (1995) Clinical and angiographic follow-up of childhood-onset moyamoya disease. Childs Nerv Syst 11:591-594

11. Ibrahimi DM, Tamargo RJ, Ahn ES (2010) Moyamoya disease in children. Childs Nerv Syst 26:1297-1308

12. Fukuyama Y, Umezu R (1985) Clinical and cerebral angiographic evolutions of idiopathic progressive occlusive disease of the circle of Willis ("moyamoya" disease) in children. Brain Dev $7: 21-37$

13. Imaizumi T, Hayashi K, Saito K, Osawa M, Fukuyama Y (1998) Long-term outcomes of pediatric moyamoya disease monitored to adulthood. Pediatr Neurol 18:321-325

14. Kurokawa T, Tomita S, Ueda K, Narazaki O, Hanai T, Hasuo K, Matsushima T, Kitamura K (1985) Prognosis of occlusive disease of the circle of Willis (moyamoya disease) in children. Pediatr Neurol 1: 274-277

15. Veeravagu A, Guzman R, Patil CG, Hou LC, Lee M, Steinberg GK (2008) Moyamoya disease in pediatric patients: outcomes of neurosurgical interventions. Neurosurg Focus 24:E16

16. Kuroda S, Ishikawa T, Houkin K, Nanba R, Hokari M, Iwasaki Y (2005) Incidence and clinical features of disease progression in adult moyamoya disease. Stroke 36:2148-2153 